\title{
Caracterização biométrica e crescimento relativo de Iphigenia brasiliensis (Lamarck, 1818) (Bivalvia: Donacidae) da Baía do Caeté, Amazônia Oriental
}

\author{
Mara Rúbia Ferreira Barros ${ }^{1,2}$ (D), Weverton John Pinheiro dos $\operatorname{Santos}^{1}$ (D), \\ Valdo Sena de Abreu ${ }^{3}$ (D), Wagner César Rosa dos Santos, ${ }^{4,5}$ (D), Marko \\ Herrmann $^{3,6}$ (D) \& Rafael Anaisce das Chagas ${ }^{1,6,7}$ (D)
}

(1) Universidade Federal Rural da Amazônia, Museu de Zoologia, Estrada Principal da UFRA 2150, Curió Utinga 66077-830, Belém, Pará, Brasil. E-mail: mail: weverton_john@hotmail.com

(2) Universidade Federal do Pará, Núcleo de Ecologia Aquática e Pesca da Amazônia, Avenida Perimetral 2651, Terra Firme 66077-530, Belém, Pará, Brasil. E-mail: eng.p.marabarros@gmail.com

(3) Universidade Federal Rural da Amazônia, Instituto Socioambiental e dos Recursos Hídricos, Programa de Pós-Graduação em Aquicultura e Recursos Aquáticos Tropicais, Avenida Presidente Tancredo Neves 2501, Curió Utinga 66077-830, Belém, Pará, Brasil.

(4) Instituto Chico Mendes de Conservação da Biodiversidade, Centro Nacional de Pesquisa e Conservação da Biodiversidade Marinha do Norte, Avenida Presidente Tancredo Neves 2501, Curió Utinga 66077-830, Belém, Pará, Brasil. E-mail: wagpesca@yahoo.com.br

(5) Museu Paraense Emílio Goeldi, Programa de Pós-Graduação em Biodiversidade e Evolução, Avenida Governador Magalhães Barata 376, Nazaré 66040-170, Belém, Pará, Brasil.

(6) Universidade Federal Rural da Amazônia, Instituto Socioambiental e dos Recursos Hídricos, Avenida Presidente Tancredo Neves 2501, Curió Utinga 66077-830, Belém, Pará, Brasil. E-mail: marko.herrmann@gmx.de

(7) Universidade Federal do Pará, Instituto de Ciências Biológicas, Programa de Pós-Graduação em Ecologia, Avenida Perimetral 2224, Guamá 66077-830, Belém, Pará, Brasil.

E-mail: rafaelanaisce@ hotmail.com

Barros M.R.F., Santos W.J.P., Abreu V.S., Santos W.C.R., Herrmann M. \& Chagas R.A. (2021) Caracterização biométrica e crescimento relativo de Iphigenia brasiliensis (Lamarck, 1818) (Bivalvia: Donacidae) da Baía do Caeté, Amazônia Oriental. Pesquisa e Ensino em Ciências Exatas e da Natureza, 5: e1837. http://dx.doi.org/10.29215/pecen.v5i0.1837

Editor acadêmico: Fabrizio Scarabino. Recebido: 11 outubro 2021. Aceito: 15 novembro 2021. Publicado: 27 dezembro 2021.

Resumo: Iphigenia brasiliensis (Lamarck, 1818) é um molusco bivalve marinho pertencente à família Donacidae, que se distribui desde o Golfo do México até o sul da costa brasileira. Habita a região intermareal, onde é coletada por marisqueiras para alimentação e comércio. O presente estudo tem o objetivo de caracterizar a biometria e o crescimento relativo de $I$. brasiliensis, coletado em um banco natural, situado na Baía do Caeté, litoral amazônico. Para tanto, mensurou-se as medidas morfométricas e determinou-se a biomassa úmida dos moluscos coletados. Posteriormente, determinou-se a correlação das variáveis e efetuaram-se relações biomorfométricas. Adicionalmente, o crescimento relativo entre as variáveis foi analisado por meio do coeficiente de alometria. Testou-se também se há relação entre as razões morfométricas da concha e a biomassa úmida dos indivíduos. Os resultados indicam que $I$. brasiliensis apresenta medidas morfométricas altamente correlacionadas, não sendo evidenciado diferenças significativas no tamanho dos indivíduos por período sazonal. Destacam-se as relações entre as medidas do comprimento anteroposterior e a altura, ambas com a biomassa úmida, como isométrica, o que indica crescimento proporcional entre essas variáveis. Além dos resultados obtidos, recomendamos estudos acerca da biologia reprodutiva e sugerimos estudos de crescimento utilizando marcadores internos para estimar o crescimento absoluto da espécie.

Palavras chave: Região Amazônica, molusco bivalve, morfometria. 
Abstract: Iphigenia brasiliensis (Lamarck, 1818) is a marine bivalve mollusk belonging to the Donacidae family, which is distributed from the Gulf of Mexico to the south of the Brazilian coast. It inhabits the intertidal region, where it is collected by shellfish gatherers for food and trade. This study aims to characterize the biometrics and relative growth of $I$. brasiliensis, collected in a natural bank, located in Baía do Caeté, Amazonian coast. For that, the morphometric measures were measured and the wet biomass of the collected molluscs was determined. Subsequently, the correlation of the variables was determined and biomorphometric relationships were performed. Additionally, the relative growth between the variables was analyzed using the allometric coefficient. It was also tested whether there is a relationship between the morphometric ratios of the shell and the wet biomass of individuals. The results indicate that I. brasiliensis has highly correlated morphometric measurements, with no significant differences in the size of individuals per seasonal period. The relationships between the measurements of anteroposterior length and height are highlighted, both with wet biomass, as isometric, which indicates proportional growth between these variables. In addition to the results obtained, we recommend studies on reproductive biology and suggest growth studies using internal markers to estimate the absolute growth of the species.

Key words: Amazon region, bivalve mollusc, morphometry.

\section{Introdução}

Os estudos acerca da caracterização biométrica de moluscos bivalves são bastante abordados na literatura (Gaspar et al. 2001, 2002; Chagas et al. 2019; Barros et al. 2020; Santos et al. 2020; Castro et al. 2021). Tais estudos possuem inúmeras aplicações ecológicas, dentre caracterizações morfológicas das conchas, auxílio na identificação taxonômica, comparações inter e intrapopulações, bem como distinção sexual (Vasconcelos \& Gaspar 2017).

O molusco bivalve Iphigenia brasiliensis (Lamarck, 1818), conhecido popularmente como "taioba” ou "tarioba" (em português), "giant false donax" (em inglês), "donace géanté" (em francês) e "coquina gigante” (em espanhol) (Leal 2002). A superfície externa de sua concha é lisa, na coloração creme bronzeado com a região umbonal na cor roxa e perióstraco marrom (nos adultos) (Leal 2002; Rios 2009; Thomé et al. 2010). Apresenta concha moderadamente inflada, em forma de cunha, pesada, com contorno romboidal (Leal 2002; Amaral et al. 2006), podendo atingir até $66.6 \mathrm{~mm}$ (Silva et al. 2012).

Iphigenia brasiliensis é uma espécie marinha pertencente à família Donacidae (Rios 2009), que possui distribuição estendendo-se desde o Golfo do México (Turgeon et al. 2009) até o Brasil, sendo no litoral brasileiro encontrados registros no estado do Amapá ao Rio Grande do Sul (Amaral et al. 2006; Thomé et al. 2010). A distribuição anterior delimitava a distribuição da espécie até o Uruguai, mas estudos recentes não registram a espécie no litoral uruguaio (Scarabino \& Zaffaroni 2004; Scarabino et al. 2016). Com isso, destaca-se que I. brasiliensis como o único donacídeo do gênero encontrado em águas brasileiras. Habita a região intermareal, sendo geralmente encontrada enterrada a $20 \mathrm{~cm}$ de profundidade em substratos inconsolidados, tais como, praias arenosas (ou areno-lodosas), em baías com influência de água doce e em estuários (Narchi 1972; Leal 2002; Amaral et al. 2006).

Mesmo apresentando importância socioeconômica ao longo da costa brasileira (com destaque aos estados de Pernambuco, Bahia e Rio de Janeiro) (Arruda et al. 2019; Ferreira et al. 2019), poucos estudos são realizados com I. brasiliensis. Algumas das pesquisas efetuadas com a espécie são: descrição dos parâmetros morfofuncionais (Narchi 1972), gametogênese (Mesquita et al. 2001; Silva et al. 2012), comportamento reprodutivo (Silva et al. 2012), casos de hermafroditismo (Ceuta et al. 2010), biometria e proporção sexual (Silva et al. 2016) e variação genética e assimetria entre populações (Bonner et al. 2019). Destaca-se também a revisão efetuada por Ferreira et al. (2019), que realizou uma revisão sistemática da bibliografia produzida a respeito de $I$. brasiliensis listando outros trabalhos encontrados sobre a espécie.

Partindo do exposto, o presente estudo tem a objetivo caracterizar biometria e o crescimento relativo de $I$. brasiliensis, coletado em um banco natural, situado na Baía do Caeté, 
região bragantina, litoral amazônico. Em seguida, testamos a hipótese de que as razões morfométricas estão correlacionadas positivamente com a biomassa úmida dos indivíduos, ou seja, quanto maior a proporção das variáveis, maior a sua biomassa.

\section{Material e Métodos}

\section{Área de coleta}

O presente estudo foi efetuado em uma área de mangue situado na Baía do Caeté, denominada Furo Grande, localizado na região bragantina, nordeste do estado do Pará (Figura 1). A região dispõe de aproximadamente $2.195 \mathrm{~km}^{2}$ de área, sendo o rio Caeté, como maior e principal rio da região, com $149 \mathrm{~km}$ de extensão. $\mathrm{O}$ índice pluviométrico anual médio de 2.100 mm, com a estação chuvosa, compreendendo os meses de dezembro a maio, e estação menos chuvosa (estação seca) correspondente ao período de junho a novembro (Moraes et al. 2005).

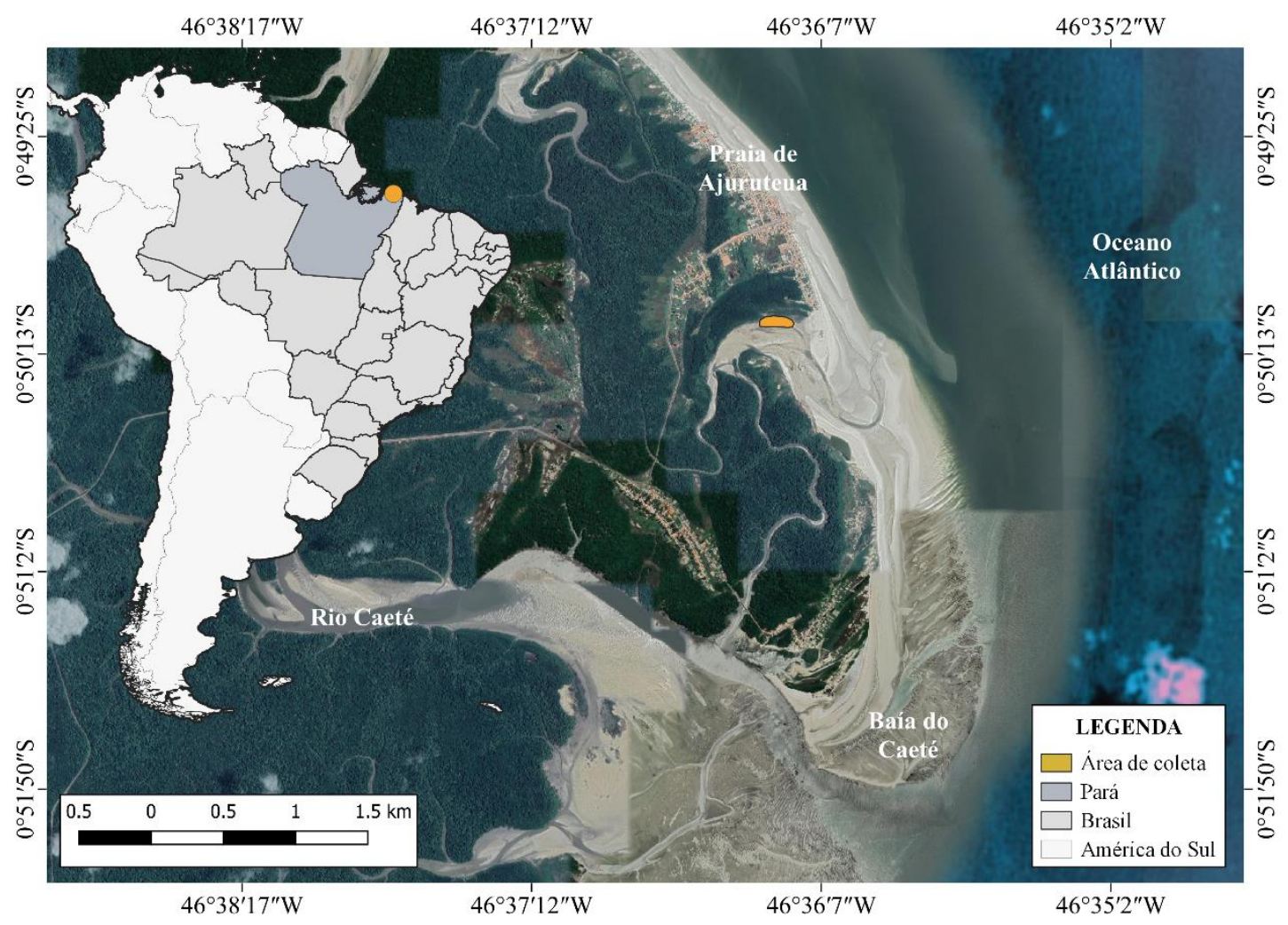

Figura 1. Localização da área de estudo, destacando a área de coleta (em laranja) de I. brasiliensis, na Baía do Caeté, litoral bragantino, Amazônia Oriental.

\section{Procedimento amostral e determinação biométrica}

Ao todo realizaram-se 11 coletas entre junho/2015 e maio/2016. A coleta de I. brasiliensis ocorreu durante marés de sizígia, utilizando-se um amostrador cilíndrico $(19 \mathrm{~cm}$ diâmetro, área $284 \mathrm{~cm}^{2}$ ), inserindo-o no substrato a uma profundidade de $15 \mathrm{~cm}$, em dez pontos aleatórios dentro da área delimitada (Figura 1). A profundidade na qual se inseriu o amostrador está de acordo com a posição da espécie no substrato (Narchi 1972). Subsequentemente fixou-se os moluscos coletados em etanol $70 \%$ e transportou-os ao laboratório de Ecologia Bentônica Tropical, localizado na Universidade Federal Rural da Amazônia - UFRA. Paralelamente mensurou-se a salinidade e temperatura superficial da água no local de coleta.

Em laboratório, mensuraram-se as medidas externas da concha (comprimento anteroposterior, largura e altura), de acordo com Leal (2002), utilizando um paquímetro digital (TESA Data-Direct, precisão de $0.01 \mathrm{~mm}$ ), em seguida, obteve-se a biomassa total úmida (Bt) dos indivíduos com auxílio de balança analítica (MH - 122 Series Pocket Scale, precisão de 0.01 g). 
Adicionalmente, calcularam-se as seguintes razões morfométricas de $I$. brasiliensis: altura/comprimento anteroposterior, altura/largura e largura/comprimento anteroposterior. Os dados brutos da biometria de $I$. brasiliensis está disponível na plataforma PANGAEA - Data Publisher for Earth \& Environmental Science (Sousa et al. 2017).

\section{Análise de dados}

Para a caracterização biométrica de $I$. brasiliensis, primeiramente testamos a existência de diferença entre as médias das medidas externas da concha, media \pm desvio padrão (DP), a biomassa úmida e as razões morfométricas por período sazonal (seco e chuvoso), utilizando um test-t. Avaliamos também a correlação entre as medidas morfométricas da concha de $I$. brasiliensis. Nossa hipótese é que a espécie apresenta medidas morfométricas altamente correlacionadas (Vasconcelos et al. 2016).

Posteriormente, efetuaram-se relações entre as medidas externas da concha utilizando equações lineares do tipo $Y=a+b \cdot X$. Efetuaram-se também relações peso/comprimento da espécie; para isso, relacionaram-se as medidas externas e a biomassa úmida, utilizando o modelo geométrico $Y=a \cdot X^{b}$. Em ambas, os coeficientes lineares (a) e angular $(b)$ significativo $(p<0.05)$ das regressões \pm desvio padrão foram estimados pelo método dos mínimos quadrados, sendo que para o modelo geométrico, utilizou-se a fórmula logarítmica: $\ln Y=\ln a+b \cdot \ln X$.

Utilizou-se o coeficiente de correlação de Pearson $\left(r^{2}\right)$ para testar a existência de dependência estatística linear entre as variáveis das relações morfométricas e de peso/comprimento. Adicionalmente, utilizou-se um test-t para verificar a significância dos valores de $r$.

O crescimento relativo entre as variáveis foi analisado por meio do coeficiente de alometria $(b)$ das relações morfométricas. Neste sentido, nas relações entre variáveis do mesmo tipo, a isometria ocorre quando $b$ não é estatisticamente diferente de 1 e nas relações entre variáveis de tipos distintos a isometria ocorre quando $b$ não é estatisticamente diferente de 3 (Vasconcelos et al. 2016). Nesses casos, significa que as taxas de crescimento de ambas as variáveis são idênticas durante a ontogenia (Huxley \& Teissier 1936). Em ambas as situações, as relações foram classificadas por alometria negativa quando $b<1$ ou $b<3$ ou alometria positiva quando $b>1$ ou $b>3$ (Vasconcelos \& Gaspar 2017).

Posteriormente, avaliamos se há relação entre as razões morfométricas da concha e a biomassa úmida dos indivíduos. Para isso, primeiramente testou-se multicolinearidade das variáveis preditoras, excluindo aquelas correlacionadas. Essa correlação foi verificada pelo Fator de Inflação da Variância (VIF), considerando valores de VIF acima de 10 como fortemente correlacionadas. Em seguida, testamos a hipótese de que as razões morfométricas estão correlacionadas positivamente com a biomassa úmida dos indivíduos, ou seja, quanto maior a proporção das variáveis, maior a sua biomassa de $I$. brasiliensis.

Para verificar a normalidade dos dados utilizou-se um teste de Shapiro-Wilk e para homogeneidade das variâncias utilizou-se um teste de Levene. Para a determinação da correlação entre as variáveis utilizou-se o pacote corrplot. A multicolinearidade foi testada utilizando o pacote car. As diferenças estatísticas foram consideradas a um nível de significância de $95 \%$ ( $\alpha=0.05$ ), utilizando o programa $\mathrm{R}$ (versão 4.1.1) ( $\mathrm{R}$ Core Team 2021) e para os gráficos utilizou-se o pacote ggplot2.

\section{Resultados e Discussão}

Ao todo foram coletados 194 indivíduos de I. brasiliensis. O menor $(5.33 \mathrm{~mm})$ e maior (44.94 mm) comprimento anteroposterior dos indivíduos, coletados em março e abril de 2016 respectivamente. A média do comprimento anteroposterior dos indivíduos foi de $21.02 \pm 8.80 \mathrm{~mm}$ (média $\pm \mathrm{DP}$ ). Um sumário dos dados mensais do comprimento anteroposterior, altura, largura e biomassa total dos indivíduos está disponível na Tabela 1. 
Tabela 1. Valores mensais (média \pm desvio padrão) do comprimento anteroposterior, largura, altura e biomassa úmida de I. brasiliensis coletados na Baía do Caeté (Pará), no período de junho/2015 a maio/2016.

\begin{tabular}{cccccc}
\hline Mês/Ano & $\mathbf{N}$ & $\begin{array}{c}\text { Comprimento } \\
\text { anteroposterior } \\
(\mathbf{m m})\end{array}$ & $\begin{array}{c}\text { Altura } \\
(\mathbf{m m})\end{array}$ & $\begin{array}{c}\text { Largura } \\
(\mathbf{m m})\end{array}$ & $\begin{array}{c}\text { Biomassa úmida } \\
(\mathrm{g})\end{array}$ \\
\hline Jun/15 & 17 & $15.47 \pm 6.88$ & $10.76 \pm 4.65$ & $4.87 \pm 2.36$ & $0.56 \pm 0.78$ \\
\hline Ago/15 & 45 & $22.60 \pm 7.58$ & $15.93 \pm 5.31$ & $7.73 \pm 2.92$ & $1.30 \pm 1.30$ \\
\hline Set/15 & 20 & $21.16 \pm 8.79$ & $14.74 \pm 5.69$ & $6.87 \pm 3.13$ & $1.13 \pm 1.37$ \\
\hline Out/15 & 3 & $9.01 \pm 2.18$ & $6.45 \pm 1.86$ & $2.74 \pm 0.88$ & $0.09 \pm 0.04$ \\
\hline Nov/15 & 30 & $21.28 \pm 10.10$ & $15.10 \pm 7.16$ & $7.07 \pm 3.74$ & $1.37 \pm 1.61$ \\
\hline Dez/15 & 12 & $24.10 \pm 8.16$ & $16.89 \pm 5.77$ & $8.15 \pm 3.11$ & $1.63 \pm 1.29$ \\
\hline Jan/16 & 2 & $7.91 \pm 0.95$ & $5.27 \pm 0.57$ & $2.34 \pm 0.40$ & $0.04 \pm 0.02$ \\
\hline Fev/16 & 12 & $16.87 \pm 10.48$ & $12.08 \pm 7.30$ & $5.35 \pm 3.67$ & $0.44 \pm 0.57$ \\
\hline Mar/16 & 6 & $10.27 \pm 4.32$ & $7.13 \pm 3.07$ & $3.20 \pm 1.42$ & $0.14 \pm 0.17$ \\
\hline Abr/16 & 16 & $26.26 \pm 7.95$ & $18.37 \pm 5.03$ & $8.71 \pm 2.82$ & $1.80 \pm 1.85$ \\
\hline Maio/16 & 30 & $23.90 \pm 5.77$ & $16.82 \pm 4.00$ & $8.05 \pm 2.18$ & $1.30 \pm 0.86$ \\
\hline
\end{tabular}

As medidas morfométricas de I. brasiliensis, especificamente comprimento, encontradas no presente estudo estão bem abaixo das encontradas em outras localidades, tais como $56.5 \mathrm{~mm}$ na Lagoa de Itaipu (Rio de Janeiro) (Mesquita et al. 2001), 50.2 no estuário do rio Subaé (Bahia) (Silva et al. 2012), $60.2 \mathrm{~mm}$ na região estuarina do Rio Cachoeira (Bahia) (Ceuta et al. 2010) e 45 mm na Baía de Guarapuá (Bahia) (Silva et al. 2016). Um dos fatores que contribui para o tamanho encontrado é o fato de que o banco natural não é predominante por I. brasiliensis e sim por Anomalocardia flexuosa (Linnaeus, 1767) (Veneridae), o que pode acarretar uma competição por alimento e espaço.

Evidencia-se que as médias das medidas externas, a biomassa úmida e as razões morfométricas da concha de $I$. brasiliensis não diferem por período sazonal (seco e chuvoso) (Tabela 2). Deste modo, refuta-se a hipótese de que os indivíduos coletados no período seco são maiores que os do período chuvoso, podendo sugerir reprodução contínua ao longo do ano para esta espécie.

Diferentemente do resultado no presente estudo, Silva et al. (2016) evidenciaram variações no tamanho e à biomassa da população de I. brasiliensis na Baía de Guarapuá (Bahia). Os autores sugerem que essa variação pode estar associada a eventos fisiológicos (p. ex., acúmulo de biomassa e período reprodutivo). No entanto, os mesmos autores citam que houve uma seletividade e limitação no processo de amostragem, o que pode ter contribuído para essa variação.

Além disso, é importante destacar a existência de variação morfométrica em moluscos bivalves sob influência latitudinal (Santos et al. 2020). Esse fator deve ser considerado em estudos populacionais, sendo um indício de que são necessários estudos pontuais em diferentes locais ao longo da ampla distribuição de uma espécie, como no caso da I. brasiliensis.

Por não haver diferença nas medidas morfométricas (comprimento anteroposterior, largura e altura) da concha de I. brasiliensis, a avalição da correlação ocorreu de modo geral, ou seja, sem separação sazonal dos dados. Os resultados corroboram a hipótese de que a espécie apresenta medidas morfométricas altamente correlacionadas (Figura 2).

A elevada correlação entre as variáveis morfométricas encontrada em I. brasiliensis no presente estudo é similar a encontrada em outras espécies de moluscos bivalves (Gaspar et al. 2001, 2002; Vasconcelos et al. 2016; Chagas et al. 2019; Barros et al. 2020). 
Tabela 2. Comparação sazonal entre as medidas morfométricas (comprimento anteroposterior, largura e altura), biomassa úmida e razões morfométricas (altura/comprimento anteroposterior, altura/largura e largura/comprimento anteroposterior) de I. brasiliensis coletados na Baía do Caeté (Pará). DP e desvio padrão.

\begin{tabular}{|c|c|c|c|c|c|c|}
\hline $\begin{array}{c}\text { Medida } \\
\text { morfométrica }\end{array}$ & Período & $\mathbf{n}$ & Média $\pm D P$ & Min.-Max. & $t$ & $p$ \\
\hline \multirow{2}{*}{$\begin{array}{l}\text { Comprimento } \\
\text { anteroposterior } \\
(\mathrm{mm})\end{array}$} & Seco & 115 & $20.59 \pm 8.77$ & $5.57-39.24$ & -0.813 & 0.417 \\
\hline & Chuvoso & 79 & $21.64 \pm 8.84$ & $5.33-44.94$ & & \\
\hline \multirow{2}{*}{ Altura (mm) } & Seco & 115 & $14.49 \pm 6.09$ & $3.93-27.82$ & -0.801 & 0.423 \\
\hline & Chuvoso & 79 & $15.21 \pm 6.10$ & $3.56-29.62$ & & \\
\hline \multirow{2}{*}{ Largura (mm) } & Seco & 115 & $6.85 \pm 3.26$ & $1.63-14.07$ & -0.694 & 0.488 \\
\hline & Chuvoso & 79 & $7.18 \pm 3.19$ & $1.45-15.03$ & & \\
\hline \multirow{2}{*}{$\begin{array}{l}\text { Biomassa } \\
\text { úmida }(\mathrm{g})\end{array}$} & Seco & 115 & $1.14 \pm 1.34$ & $0.01-5.75$ & -0.187 & 0.851 \\
\hline & Chuvoso & 79 & $1.18 \pm 1.24$ & $0.01-7.96$ & & \\
\hline \multirow{2}{*}{$\begin{array}{c}\text { Altura / } \\
\text { Comprimento } \\
\text { anteroposterior }\end{array}$} & Seco & 115 & $0.70 \pm 0.03$ & $0.65-0.84$ & 0.371 & 0.710 \\
\hline & Chuvoso & 79 & $0.70 \pm 0.03$ & $0.64-0.88$ & & \\
\hline \multirow{2}{*}{$\begin{array}{l}\text { Altura / } \\
\text { Largura }\end{array}$} & Seco & 115 & $2.18 \pm 0.20$ & $1.81-3.13$ & -0.102 & 0.918 \\
\hline & Chuvoso & 79 & $2.18 \pm 0.23$ & $1.89-3.28$ & & \\
\hline \multirow{2}{*}{$\begin{array}{c}\text { Largura / } \\
\text { Comprimento } \\
\text { anteroposterior }\end{array}$} & Seco & 115 & $0.32 \pm 0.02$ & $0.26-0.38$ & 0.247 & 0.805 \\
\hline & Chuvoso & 79 & $0.32 \pm 0.02$ & $0.23-0.37$ & & \\
\hline
\end{tabular}

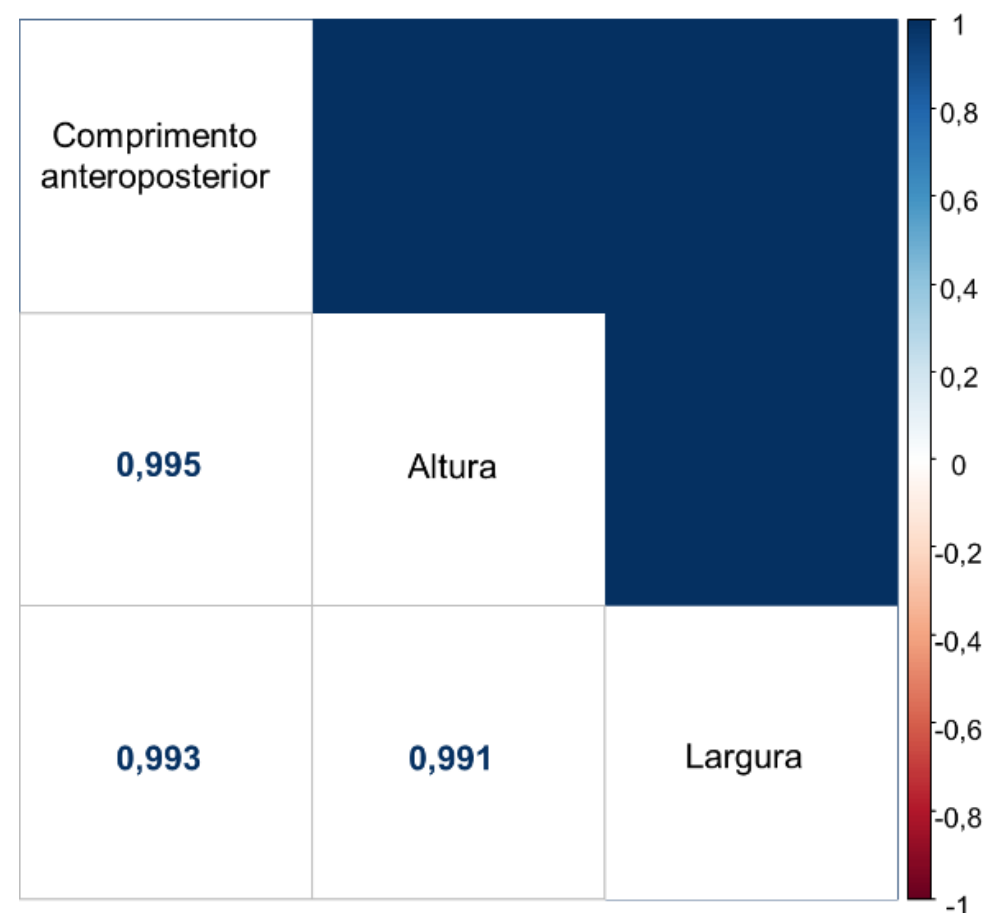

Figura 2. Correlação entre as medidas morfométricas da concha de I. brasiliensis, coletada na Baía do Caeté, litoral bragantino, Amazônia Oriental.

Assim como a avaliação da correlação, as relações biomorfométricas entre as medidas externas da concha e a biomassa úmida de I. brasiliensis foram realizados de modo geral, sem distinção por período (Tabela 3). Isso significa também que não se evidencia diferença no crescimento relativo da espécie por influência sazonal. Os coeficientes de correlação de Pearson 
indicam que todas as relações foram altamente significativas $\left(r^{2}>0.9 ; p<0.001\right)$. As relações morfométricas envolvendo o comprimento anteroposterior foram classificadas por alometria negativa, o que indica que esta medida morfométrica cresce em uma taxa maior do que as demais. De altura e largura classificada por alometria positiva, indicando que a altura da espécie cresce em uma taxa superior a largura.

Tabela 3. Relações entre as medidas morfométricas (comprimento anteroposterior, largura e altura) e entre as medidas morfométricas e a biomassa úmida de $I$. brasiliensis coletados na Baía do Caeté (Pará). Legenda: ** significam valores de $p<0.001$.

\begin{tabular}{ccccc}
\hline Relações & $\mathrm{a} \pm \mathrm{DP}$ & $\mathrm{b} \pm \mathrm{DP}$ & $\mathrm{r}^{2}$ & Alometria \\
\hline Altura $=a+b$ Comprimento & $0.30 \pm 0.11^{* *}$ & $0.68 \pm 0.01^{* *}$ & 0.989 & Negativa \\
\hline Largura $=a+b$ Comprimento & $-0.66 \pm 0.07^{* *}$ & $0.36 \pm 0.01^{* *}$ & 0.985 & Negativa \\
\hline Altura $=a+b$ Largura & $1.70 \pm 0.13^{* *}$ & $1.87 \pm 0.01^{* *}$ & 0.983 & Positiva \\
\hline $\log$ Biomassa $=\log a+b \cdot \log$ Comprimento & $-9.73 \pm 0.16^{* *}$ & $3.08 \pm 0.05^{* *}$ & 0.942 & Isométrica \\
\hline $\log$ Biomassa $=\log a+b \cdot \log$ Largura & $-5.64 \pm 0.08^{* *}$ & $2.73 \pm 0.04^{* *}$ & 0.948 & Negativa \\
\hline $\log$ Biomassa $=\log a+b \cdot \log$ Altura & $-8.68 \pm 0.15^{* *}$ & $3.09 \pm 0.05^{* *}$ & 0.934 & Isométrica \\
\hline
\end{tabular}

As relações entre as medidas do comprimento anteroposterior e a altura, ambas com a biomassa úmida, foram classificadas como isométrica. Esse resultado indica que essas medidas morfométricas apresentam crescimento proporcional ao longo do desenvolvimento da espécie. Já a relação entre a largura da concha e a biomassa úmida foi classificada por alometria negativa, o que indica que a largura da concha apresenta crescimento em uma taxa maior que a biomassa úmida da espécie.

Por fim, o teste de multicolinearidade das variáveis preditoras, indicou que as razões morfométricas da concha de I. brasiliensis são fortemente correlacionadas. Deste modo, utilizouse apenas uma das razões morfométricas para testar a hipótese de correlação positivamente com a biomassa úmida dos indivíduos. Os resultados refutam a hipótese de que quanto maior a proporção das variáveis, maior a sua biomassa de $I$. brasiliensis. Sendo assim, evidencia-se que as razões morfométricas e a biomassa úmida da espécie apresentam uma baixa correlação negativa, com tendência a nulidade $\left(r^{2}=0.022 ; p=0.020\right)$ (Figura 3 ).

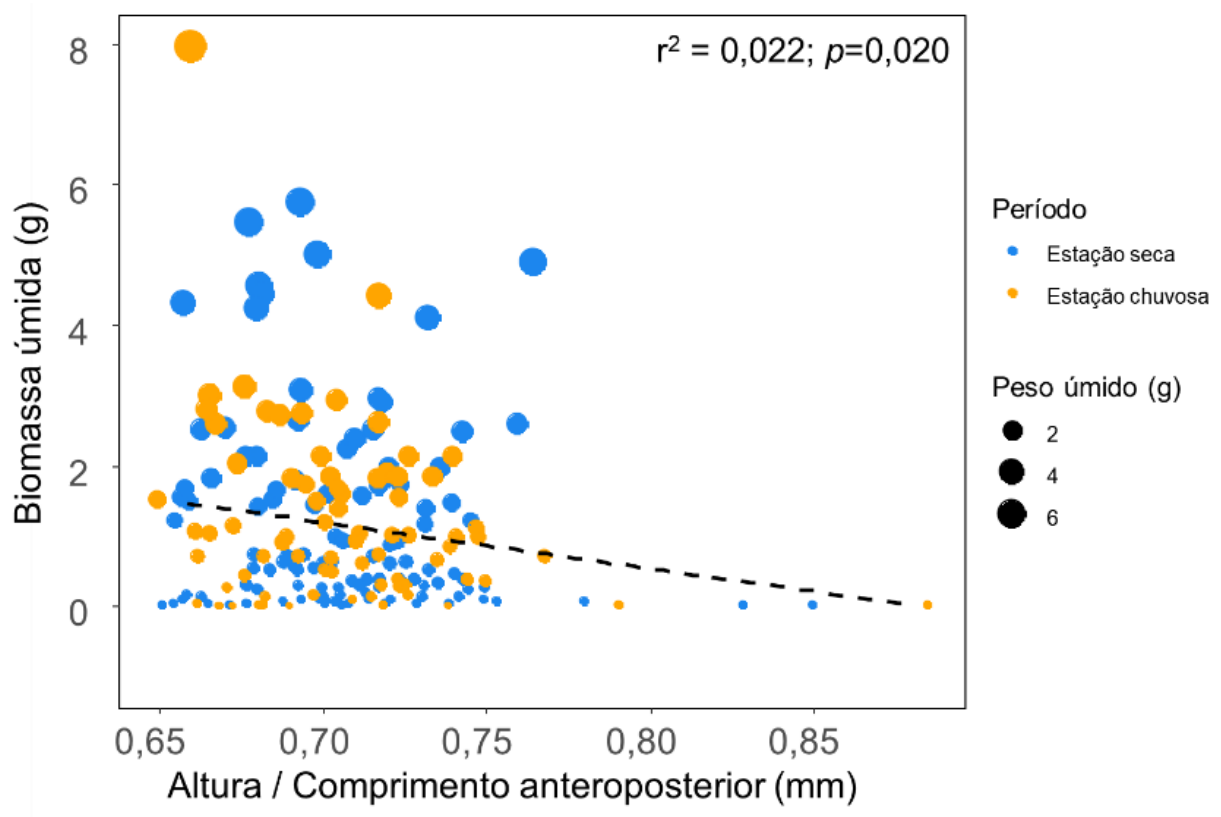

Figura 3. Relação entre as razões morfométricas e a biomassa úmida da concha de I. brasiliensis, coletada na Baía do Caeté, litoral bragantino, Amazônia Oriental. 
O estabelecimento das relações morfométricas da espécie possibilitam a formulação de equações que podem ser utilizadas para a conversão das medidas morfométricas (Vasconcelos \& Gaspar 2017), o que possibilita também determinar a estimação de rendimento da carne (Chagas et al. 2019; Barros et al. 2020) ou aplicada em estudos taxonômicos de moluscos (Altomari et al. 2021a-b).

Ao analisar o crescimento de moluscos bivalves, o ideal é realizar um experimento de marcação-recaptura para assim poder mensurar o crescimento absoluto da espécie (Gosling 2015). Nestes casos, principalmente por tratar-se de uma espécie de clima tropical, é recomendado a utilização de marcadores internos (p. ex., fluorocromo calceína) (Herrmann et al. 2009; Lepore et al. 2009; Chagas \& Herrmann 2016), o que permite a determinação do crescimento absoluto de modo mais eficaz (Chagas et al. 2020).

Os bivalves são acometidos por inúmeros fatores abióticos e bióticos, o que contribuem para a alta plasticidade fenotípica das espécies (Vasconcelos et al. 2016). Neste sentido, estudos biométricos são importantes para subsidiar ações conservacionistas para espécies com importância socioeconômica, como a I. brasiliensis e assim, possibilitando a gestão extrativista do molusco (Silva et al. 2016).

\section{Conclusão}

Neste estudo caracterizamos a biometria e o crescimento relativo do molusco bivalve Iphigenia brasiliensis coletados na Baía do Caeté, concluindo que a espécie apresenta medidas morfométricas altamente correlacionadas nesta área. Além disso, não foram evidenciadas diferenças significativas no tamanho dos indivíduos por período sazonal, o que indica a presença de várias coortes e, consequentemente, que a espécie apresenta comportamento de reprodução contínuo. Destacam-se as relações entre as medidas do comprimento anteroposterior e a altura, ambas com a biomassa úmida, como isométrica, o que indica crescimento proporcional entre essas variáveis.

Devido ao período curto no presente estudo, e à ausência de análise especificadamente voltada ao ciclo reprodutivo da espécie, recomendamos um estudo aprofundado e usando metodologia adequada para testar a existência de um ciclo reprodutivo contínuo, evidenciado neste estudo. Paralelamente, sugerimos estudos de crescimento utilizando marcadores internos para estimar o crescimento absoluto da espécie.

Além disso, devido à existência de uma atividade extrativista e comercial da espécie, ressaltamos a necessidade de estudos voltados a recomendações de tamanho mínimo de captura e/ou períodos permitidos de extração. Tais informações, adicionados a estudos ecológicos, de dinâmica populacional e de crescimento dos organismos, tal como este, que abordem a caracterização biométrica e o crescimento relativo, são de vital importância, pois subsidiam ações à conservação e possibilitam a gestão para o manejo sustentável da espécie.

\section{Agradecimentos}

Os autores deste trabalho agradecem ao Instituto Socioambiental e dos Recursos Hídricos (ISARH) da Universidade Federal Rural da Amazônica (UFRA), pelo apoio logístico para a realização das amostragens de campo. Aos alunos do curso de Engenharia de Pesca (UFRA) que participaram das amostragens de campo. Ao Programa de Pós-Graduação em Ecologia (PPGEco/UFPA), ao Programa de Pós-Graduação em Ecologia Aquática e Pesca (PPGEAP/UFPA) e ao Programa de Pós-Graduação em Aquicultura e Recursos Aquáticos Tropicais (PPGAqRAT/UFRA), em nome dos autores RA Chagas, MRF Barros e VS Abreu, respectivamente. A Coordenação de Aperfeiçoamento de Pessoal de Nível Superior - CAPES pelas bolsas de pesquisa concedidas aos autores mencionados anteriormente. E aos revisores pelos comentários e revisões críticas que possibilitaram uma melhor adequação e escrita deste artigo. 


\section{Referências}

Altomari L.N., Alves B.H.B., Santos W.J.P., Barros M.R.F., Herrmann M., Bezerra A.M. \& Chagas R.A. (2021a) Shell morphometric ratios as a tool for taxonomic determination in gastropods: a case study in Nerita (Gastropoda, Neritidae). Journal of the Marine Biological Association of the United Kingdom, 101(4): 1-7. https://doi.org/10.1017/S0025315421000643

Altomari L.N., Alves B.H.B., Santos W.J.P., Santos W.C.R., Barros M.R.F., Bezerra A.M., Herrmann M. \& Chagas R.A. (2021b) Conquiliometria de gastrópodes neritídeos da praia de Accra, Barbados, Mar do Caribe. Acta of Fisheries and Aquatic Resources, 9(1): 1-6. https://doi.org/10.46732/actafish.2021.9.1.1-6

Amaral A.C.Z., Rizzo A.E. \& Arruda E.P. (2006) Manual de identificação dos invertebrados marinhos da região Sudeste-Sul do Brasil. São Paulo: Editora da Universidade de São Paulo. $288 \mathrm{p}$.

Arruda T.A., Duarte M.R., Souza R.C.C.L., Gomes A.S. \& Silva E.P. (2019) Zooarqueología de los restos malacológicos de Sambaqui da Tarioba (Rio das Ostras-RJ, Brasil). Archaeofauna, 28 : 95-104. https://doi.org/10.15366/archaeofauna2019.28.008

Barros M.R.F., Santos W.J.P. \& Chagas R.A. (2020) Morphometry and shell shape stabilization indicator (IEF) of the mussel Mytella charruana (d'Orbigny, 1842) (Bivalvia, Mytilidae). Biota Amazônia, 10(1): 31-34. http://dx.doi.org/10.18561/2179-5746/biotaamazonia.v10n1p31-34

Bonner A., Ferreira M.S.N., Duarte M.R. \& Silva E.P. (2019) Genetic variation and asymmetry in populations of Iphigenia brasiliensis (Lamarck, 1818) from different localities and environments. Journal of Natural History, 53(1-2): 73-88. http://dx.doi.org/10.1080/00222933.2019.1576934

Castro L.R., Santos W.J.P., Barros M.R.F., Bezerra A.M., Herrmann M. \& Chagas R.A. (2021) Estabilização conquiliométrica em moluscos: estudo de caso com Sphenia fragilis (H. Adams \& A. Adams, 1854) (Bivalvia, Myidae). Research, Society and Development, 10(3): e23510313125. https://doi.org/10.33448/rsd-v10i3.13125

Ceuta L.O., Boehs G. \& Santos J.J.B. (2010) Hermaphroditism among dioecious Tagelus plebeius (Lightfoot, 1786) (Mollusca, Psammobiidae) and Iphigenia brasiliana (Lamarck, 1818) (Mollusca, Donacidae) on the Cachoeira River Estuary, Ilhéus, Bahia, Brazil. Brazilian Journal of Biology, 70: 125-127.

Chagas R.A., Barros M.R.F., Santos W.C.R., Brabo M.F. \& Herrmann M. (2020) Uso de métodos tradicionais e científicos em pesquisas envolvendo crescimento de ostras. Acta of Fisheries and Aquatic Resources, 8(1): 24-28. http://dx.doi.org/10.46732/actafish.2020.8.1.24-28

Chagas R.A. \& Herrmann M. (2016) Estimativas de crescimento de bivalves tropicais e subtropicais: recomendação para um método padronizado. Acta of Fisheries and Aquatic Resources, 4(2): 28-38. https://doi.org/10.2312/ActaFish.4016.4.2.28-38

Chagas R.A., Silva R.E.O., Passos T.A.F., Assis A.S., Abreu V.S., Santos W.C.R., Barros M.R.F. \& Herrmann M. (2019) Análise biomorfométrica da ostra-do-mangue cultivada no litoral amazônico. Scientia Plena, 15(10): 1-13. http://dx.doi.org/10.14808/sci.plena.2019.107401

Ferreira M.S.N., Duarte M.R. \& Silva E.P. (2019) Recursos Pesqueiros de Mariscagem: uma Revisão da Literatura sobre Iphigenia brasiliensis. Ensaios e Ciência: Ciências Biológicas, Agrárias e da Saúde, 23(1): 24. http://dx.doi.org/10.17921/1415-6938.2019v23n1p24-34

Gaspar M.B., Santos M.N. \& Vasconcelos P. (2001) Weight-length relationships of 25 bivalve species (Mollusca: Bivalvia) from the Algarve coast (southern Portugal). Journal of the Marine Biological Association of the United Kingdom, 81: 805-807. https://doi.org/10.1017/S0025315401004623

Gaspar M.B., Santos M.N., Vasconcelos P. \& Monteiro C.C. (2002) Shell morphometric relationships of the most common bivalve species (Mollusca: Bivalvia) of the Algarve coast (southern Portugal). Hydrobiologia, 477: 73-80. https://doi.org/10.1023/A:1021009031717

Gosling E. (2015) Marine Bivalve Molluscs. $2^{\circ}$ edition. New Jersey: John Wiley \& Sons, Ltd. 537 p.

Herrmann M., Lepore M.L., Laudien J., Arntz W.E. \& Penchaszadeh P.E. (2009) Growth estimations of the Argentinean wedge clam Donax hanleyanus: A comparison between 
length-frequency distribution and size-increment analysis. Journal of Experimental Marine Biology and Ecology, 379(1-2): 8-15. https://doi.org/10.1016/j.jembe.2009.07.031

Huxley J.S. \& Teissier G. (1936) Terminology of Relative Growth. Nature, 137: 780-781. https://doi.org/10.1038/137780b0

Leal J.H. (2002) Bivalves (p. 25-98). In: Carpenter K.E. (ed.) The Living Marine Resources of the Western Central Atlantic. Volume 1: Introduction, molluscs, crustaceans, hagfishes, sharks, batoid fishes and chimaeras. FAO: Rome. $600 \mathrm{p}$.

Lepore M.L., Penchaszadeh P.E., Alfaya J.E.F. \& Herrmann M. (2009) Aplicación de calceína para la estimación del crecimiento de la almeja amarilla Mesodesma mactroides Reeve, 1854. Revista de Biologia Marina y Oceanografia, 44(3): 767-774. https://doi.org/10.4067/S0718-19572009000300022

Mesquita E.F.M., Abreu M.G. \& Lima F.C. (2001) Aspectos gametogênicos e histoquímicos de Iphigenia brasiliana (Lamarck) (Bivalvia, Donacidae) da Lagoa de Itaipu, Niterói, Rio de Janeiro, Brasil. Revista Brasileira de Zoologia, 18(2): 523-528.

Moraes B.C., Costa J.M.N., Costa A.C.L. \& Costa M.H. (2005) Variação espacial e temporal da precipitação no estado do Pará. Acta Amazonica, 35(2): 207-214. http://dx.doi.org/10.1590/S0044-59672005000200010

Narchi W. (1972) On the biology of Iphigenia brasiliensis Lamarck, 1818 (Bivalvia, Donacidae). Proceedings Malacological Society of London, 40: 79-91. https://doi.org/10.1093/oxfordjournals.mollus.a065213

R Core Team (2021) R: A language and environment for statistical computing. R Foundation for Statistical Computing.

Rios E.C. (2009) Compendium of Brazilian Sea Shells. Rio Grande, RS: Evangraf. 676 p.

Santos W.J.P., Melo A.C., Gomes A.C.A., Barros M.R.F., Chagas R.A. \& Bezerra A.M. (2020) Variação morfométrica de Tivela mactroides (Bivalvia, Veneridae) no litoral Norte-Nordeste do Brasil. Revista Brasileira de Zoociências, 21(1): 1-11.

Scarabino F. \& Zaffaroni J.C. (2004) Estatus faunístico de veinte especies de moluscos citadas para aguas uruguayas. Comunicaciones Zoológicas, Museo Nacional de Historia Natural y Antropología, 13(202): 1-16.

Scarabino F., Zelaya D.G., Orensanz J.M.L., Ortega L., Defeo O., Schwindt E., Carranza A., Zaffaroni J.C., Martínez G., Scarabino V. \& García-Rodríguez F. (2016) Cold, warm, temperate and brackish: Bivalve biodiversity in a complex oceanographic scenario (Uruguay, southwestern Atlantic). American Malacological Bulletin, 32(2): 284-301. https://doi.org/10.4003/006.033.0219

Silva P.P., Peso-Aguiar M.C. \& Paz J.R.L. (2016) Biometria e proporção sexual de Iphigenia brasiliana (Lamarck, 1818) (Bivalvia, Donacidae) da baía de Guarapuá, Cairu, BA. Arquivo de Ciências do Mar, 49(2): 7-14. https://doi.org/10.32360/acmar.v49i2.6551

Silva P.P., Peso-Aguiar M.C. \& Ribeiro G. (2012) Ciclo gametogênico e comportamento reprodutivo de Iphigenia brasiliana (Mollusca, Bivalvia, Donacidae) no estuário do rio Subaé, Baía de todos os Santos, Bahia, Brasil. Iheringia, 102(4): 359-369.

Sousa T.C.L., Abreu V.S., Silva R.E.O., Barros M.R.F., Chagas R.A. \& Herrmann M. (2017) Alometry and relative growth of Iphigenia brasiliensis (Lamarck, 1818) (Bivalvia: Donacidae) in Caeté Bay, Pará, Brazil. PANGAEA - Data Publisher for Earth \& Environmental Science. https://doi.org/10.1594/PANGAEA.879485

Thomé J.W., Gil G.M., Bergonci P.E.A. \& Tarasconi J.C. (2010) As conchas das nossas praias. $2^{\circ}$ edição. Porto Alegre: Redes Editora. $224 \mathrm{p}$.

Turgeon D.D., Lyons W.G., Mikkelsen P., Rosenberg G. \& Moretzsohn F. (2009) Bivalvia (Mollusca) of the Gulf of Mexico (p. 711-744). In: Felder D.L. \& Camp D.K. (ed.) Gulf of Mexico: origin, waters, and biota. Volume 1: Biodiversity. Texas: Texas A\&M University Press. $1393 \mathrm{p}$.

Vasconcelos P. \& Gaspar M. (2017) A importância e utilidade dos estudos morfométricos e do crescimento relativo em bivalves e gastrópodes. Portugala, 20: 10-11. 
Vasconcelos P., Moura P., Pereira F., Pereira A.M. \& Gaspar M.B. (2016) Morphometric relationships and relative growth of 20 uncommon bivalve species from the Algarve coast (southern Portugal). Journal of the Marine Biological Association of the United Kingdom, 98(3): 463-474. https://doi.org/10.1017/s002531541600165x 